\title{
Mesenchymal Stromal Cells Accelerate Epithelial Tight Junction Assembly via the AMP-Activated Protein Kinase Pathway, Independently of Liver Kinase B 1
}

\author{
P. Rowart, ${ }^{1}$ P. Erpicum, ${ }^{1,2}$ J.-M. Krzesinski, ${ }^{1,2}$ M. Sebbagh, ${ }^{3}$ and F. Jouret ${ }^{1,2}$ \\ ${ }^{1}$ Groupe Interdisciplinaire de Génoprotéomique Appliquée (GIGA), Cardiovascular Sciences, University of Liège (ULg), \\ Liège, Belgium \\ ${ }^{2}$ Division of Nephrology, University of Liège Hospital (ULg CHU), Liège, Belgium \\ ${ }^{3}$ Centre de Recherche en Cancérologie de Marseille, Aix Marseille Université UM105, Institut Paoli Calmettes, UMR7258 CNRS, \\ U1068 INSERM, Cell Polarity, Cell Signalling and Cancer "Equipe Labellisée Ligue Contre le Cancer", Marseille, France
}

Correspondence should be addressed to F. Jouret; francois.jouret@ulg.ac.be

Received 30 March 2017; Accepted 21 May 2017; Published 11 July 2017

Academic Editor: Bruno Christ

Copyright ( $\odot 2017$ P. Rowart et al. This is an open access article distributed under the Creative Commons Attribution License, which permits unrestricted use, distribution, and reproduction in any medium, provided the original work is properly cited.

\begin{abstract}
Background. Mesenchymal stromal cells (MSC) are fibroblast-like multipotent cells capable of tissue-repair properties. Given the essentiality of tight junctions (TJ) in epithelial integrity, we hypothesized that MSC modulate TJ formation, via the AMP-activated kinase (AMPK) pathway. Liver kinase- $\beta 1$ (LKB1) and $\mathrm{Ca}^{2+}$-calmodulin-dependent protein kinase kinase (CaMKK) represent the main kinases that activate AMPK. Methods. The in vitro $\mathrm{Ca}^{2+}$ switch from $5 \mu \mathrm{M}$ to $1.8 \mathrm{mM}$ was performed using epithelial MadinDarby canine kidney (MDCK) cells cultured alone or cocultured with rat bone marrow-derived MSC or preexposed to MSCconditioned medium. TJ assembly was measured by assessing ZO-1 relocation to cell-cell contacts. Experiments were conducted using MDCK stably expressing short-hairpin-RNA (shRNA) against LKB1 or luciferase (LUC, as controls). Compound STO-609 $(50 \mu \mathrm{M})$ was used as CaMKK inhibitor. Results. Following $\mathrm{Ca}^{2+}$ switch, ZO-1 relocation and phosphorylation/activation of AMPK were significantly higher in MDCK/MSC compared to MDCK. No difference in AMPK phosphorylation was observed between LKB1-shRNA and Luc-shRNA MDCK following $\mathrm{Ca}^{2+}$ switch. Conversely, incubation with STO-609 prior to $\mathrm{Ca}^{2+}$ switch prevented AMPK phosphorylation and ZO-1 relocation. MSC-conditioned medium slightly but significantly increased AMPK activation and accelerated TJ-associated distribution of $\mathrm{ZO}-1$ post $\mathrm{Ca}^{2+}$ switch in comparison to regular medium. Conclusions. MSC modulate the assembly of epithelial TJ, via the CaMKK/AMPK pathway independently of LKB1.
\end{abstract}

\section{Introduction}

Epithelial tight junctions (TJ) form a seal at the superior pole of the lateral plasma membrane when cells differentiate and acquire polarity [1]. TJ regulate the passage of ions and small molecules through the paracellular pathway [2] and also restrict the diffusion of membrane proteins between the apical and basolateral compartments. TJ are made of at least 40 different proteins including transmembrane proteins, such as claudins and occludins, and adaptor proteins, such as members of the MAGUK (membrane-associated guanylate kinase) family, ZO-1, ZO-2, and ZO-3 [3, 4]. At the time of TJ assembly, ZO-1 and ZO-2 have essential roles in both organizing $\mathrm{TJ}$ components and targeting them to their proper location [5]. Many factors have been identified as modulators of $\mathrm{TJ}$ assembly/disassembly, including extracellular $\mathrm{Ca}^{2+}[6]$. Extracellular $\mathrm{Ca}^{2+}$ is essential for both the development of new junctions [7] and the stabilization of mature junctions $[8,9]$ between epithelial cells [10]. The dependence of TJ assembly on $\mathrm{Ca}^{2+}$ is probably attributable to the stabilization of the cell adhesion molecule E-cadherin in its adhesive state. Numerous pathways have been implicated in TJ regulation, including the AMP-activated protein kinase [1,11-16].

AMPK is an ubiquitous heterotrimeric complex made of 1 catalytic $\alpha$-subunit and 2 regulatory $\beta$ - and $\gamma$-subunits 
[17]. AMPK activity is modulated by the intracellular AMP-to-ATP ratio, as well as by the activity of upstream AMPK kinases, such as liver kinase- $\beta 1$ (LKB1) and $\mathrm{Ca}^{2+}$-calmodulin-dependent protein kinase kinase (CaMKK) [18-20]. An increased ratio of AMP/ATP induces AMP binding to the $\gamma$-subunit, thereby promoting AMPK phosphorylation at a threonine residue (Thr-172) and its activation [21]. Additionally, in case of energy stress, LKB1 phosphorylates and activates AMPK via the formation of a complex with the pseudokinase STRAD and the scaffolding protein MO25 [22]. CaMKK activates AMPK in an AMP-independent manner in response to increased cytosolic calcium concentration $[23,24]$. Note that AMPK autophosphorylation at $\beta$-subunit Thr-148 has been reported [25]. Activated AMPK promotes ATP production by favoring catabolism and switching off anabolic pathways. Interestingly, the pharmacological activation of AMPK by AICAR induces TJ assembly, independently of extracellular $\mathrm{Ca}^{2+}$ or energy deprivation [11, 18]. This effect might be achieved by strengthening the trans interactions mediated by cell adhesion molecules involved in the nectin-I-afadin system $[12,26]$ and/ or by modulating cytoskeleton dynamics near the cell membrane [12]. Furthermore, preactivation of AMPK by metformin or AICAR helps preserve the functional integrity of epithelial cells in the face of ischemia and energy depletion, as demonstrated in vitro and in vivo [27-29]. TJ disruption is indeed considered as one of the earliest hallmarks of epithelial injury, leading to the loss of cell polarity and tissue disorganization.

Cumulative evidence in the field of epithelial injury supports that mesenchymal stromal cells (MSC) are capable of tissue-repair properties [30]. MSC represent a heterogeneous population of adult fibroblast-like multipotent cells [31]. In addition to their beneficial immunomodulatory and antiinflammatory abilities [32], MSC may help epithelial cells survive, proliferate, and differentiate following injury [30, 33-35]. Hence, recent in vitro observations highlighted the role of MSC in wound healing of airway epithelium, via direct cell-cell contacts and paracrine activation of the epidermal growth factor receptor [36-38]. Also, MSC are known to release membrane vesicles (MVs) of various size and composition into the extracellular environment [39]. MSC-derived MVs may help transfer cytosolic components, including proteins, lipids, RNA, and organelles, from MSC to neighboring cells, which accelerate tissue repair $[40,41]$.

In the present study, we first investigated which AMPK kinases were responsible for AMPK phosphorylation and activation at the time of a $\mathrm{Ca}^{2+}$ switch. Next, we questioned the impact of MSC on epithelial TJ regulation in a coculture system of $\mathrm{Ca}^{2+}$-induced TJ assembly in MDCK cells. Finally, we studied the impact of MSC-conditioned medium on epithelial TJ assembly. This paper was presented at MiSOT 2016-The 6th Expert Meeting on Therapeutic MSCs for Immune Modulation.

\section{Materials and Methods}

2.1. MDCK Culture Conditions. MDCK cells were grown to confluence in $\alpha$-MEM supplemented with $10 \%$ FBS, $1 \% \mathrm{~L}$ - glutamine (Lonza), and $1 \%$ penicillin (Lonza), in a humidified atmosphere containing $5 \% \mathrm{CO}_{2}$ at $37^{\circ} \mathrm{C}$. MDCK shRNA for LKB1 and luciferase ( $L u c$, used as controls) were generated using pSUPER/retro-puro vector, as previously reported [24]. Stable populations were maintained using puromycin ( $2 \mu \mathrm{g} / \mathrm{mL}$; Sigma) as selection agent.

2.2. Isolation and Characterization of Bone Marrow-Derived MSC. Bone marrow cells were flushed from both femurs and tibias of male 9-week-old Lewis rats using phosphatebuffered saline (PBS, Lonza). After homogenization, cell suspension was filtered and centrifuged at $1200 \mathrm{rpm}$ for $10 \mathrm{~min}$. Cells were resuspended in $\alpha$-MEM medium (Lonza) and gently sieved through Ficoll (Healthcare Life Sciences). After an additional $1500 \mathrm{rpm}$ centrifugation for $45 \mathrm{~min}$ at room temperature (RT), mononuclear cells were removed from the gradient interface and suspended in $\alpha$-MEM solution before final $1200 \mathrm{rpm}$ centrifugation for $10 \mathrm{~min}$. The cells were then plated in $75 \mathrm{~cm}^{2}$ culture flask containing $\alpha$-MEM supplemented with $10 \%$ FBS, $1 \%$ L-glutamine, and $1 \%$ penicillin. MSC were maintained at $37^{\circ} \mathrm{C}$ in a humidified $5 \% \mathrm{CO}_{2}$ incubator. Supplemented $\alpha$-MEM was changed twice a week. Cells were trypsinised at $80 \%$ of confluence for maximum 8 passages. At confluence, fresh culture medium was poured, collected at day 3, centrifuged at $1.800 \mathrm{rpm}$ for $5 \mathrm{~min}$, and stored at $-80^{\circ} \mathrm{C}$ for further use. MSC phenotype was tested according to the criteria of the International Society of Cell Therapy: (i) plastic adherence, (ii) (non)expression of conventional surface markers using flow cytometry; and (iii) differentiation into adipogenic, osteogenic, and chondrogenic lineages [42]. Flow cytometry was performed on a FACSCalibur flow cytometer (BD Biosciences), using Alexa Fluorconjugated anti-rat CD29 antibody (BD Pharmingen), APC-conjugated anti-rat CD90 antibody (BD Pharmingen), V450-conjugated anti-rat CD45 antibody (BD Horizon), FITC-conjugated anti-rat CD11b (BD Pharmingen), and PE-conjugated anti-CD79a antibody (Abcam).

2.3. MSC/MDCK Coculture System. Cell populations were mixed and seeded on 6-well plates at density $1.5 \times 10^{5}$ cells/ well. The seeding ratio of MSC:MDCK was $1: 3$. All experiments were performed at confluence. Alternatively, MDCK cells were incubated with $2 \mathrm{~mL}$ of $\alpha$-MEM medium preexposed to MSC for 3 days.

2.4. $\mathrm{Ca}^{2+}$ Switch Experiments. Following steady state, cells were rinsed and incubated in $\mathrm{Ca}^{2+}$-free S-MEM supplemented with $5 \%$ dialyzed FBS $\left(\left[\mathrm{Ca}^{2+}\right], 5 \mu \mathrm{M}\right)$ for $16 \mathrm{~h}$ before being switched back to normal medium $\left(\alpha-\mathrm{MEM} ;\left[\mathrm{Ca}^{2+}\right]\right.$, $1.8 \mathrm{mM})$ for the indicated times. Compounds STO-609 (50 $\mu \mathrm{M}$; Sigma) and dorsomorphin/compound C $(50 \mu \mathrm{M}$; Sigma) were used as CaMKK and AMPK inhibitors, respectively. Increasing concentrations of dorsomorphin/compound $\mathrm{C}$ were tested in order to fully block AMPK activation (Supplementary Figure, panel A, available online at https://doi.org/10.1155/2017/9717353). Alternatively, MDCK cells were exposed to MSC-conditioned medium for $24 \mathrm{~h}$, before exposure to S-MEM for $16 \mathrm{~h}$. The $\mathrm{Ca}^{2+}$ switch 
was then realized using MSC-conditioned $\alpha$-MEM for the indicated times.

2.5. Western Blot Analysis. Cells were lysed on ice in RIPA lysis buffer including protease and phosphatase inhibitors (Roche). Cell lysates were obtained by centrifugation at $13.000 \mathrm{rpm}$ for $30 \mathrm{~min}$ at $4^{\circ} \mathrm{C}$. Supernatant was collected. Protein concentration was determined using Bradford method. Protein lysates were mixed with Laemmli buffer $(1: 4)$ and heated for $2 \mathrm{~min}$ at $95^{\circ} \mathrm{C}$. Equal amounts of protein $(30 \mu \mathrm{g} /$ lane) were loaded onto stain-free SDS electrophoresis gels and separated at $100 \mathrm{~V}$ (Bio-Rad). Gels were exposed to UV light for $5 \mathrm{~min}$ (ChemiDoc MP system, Bio-Rad). Proteins were transferred to PVDF membranes (previously activated by ethanol) using the Trans-Blot Turbo transfer system for 7 min at RT. Blots were blocked with $5 \%$ milk in Trisbuffered saline with Tween 20 (TBS-T) for $1 \mathrm{~h}$ and incubated overnight at $4^{\circ} \mathrm{C}$ with primary antibodies: pAMPK (T172) (Cell Signaling), AMPK (Cell Signaling), and pACC (Cell Signaling) antibodies. Blots were rinsing 5 times with TBS$\mathrm{T}$ for $5 \mathrm{~min}$ and incubated with HRP-conjugated anti-rabbit secondary antibodies $(1 / 4000)$ for $90 \mathrm{~min}$ at RT. After rinsing, chemiluminescent signals were captured by ChemiDoc MP system after applying chemiluminescent substrate (Femto, Thermoscientific) on blots. Image data were analyzed and quantified ( $n=4$ for each experimental condition) using Image Lab 4.1 software. Representative samples were then run on the same stain-free SDS gels for the sake of publication, in agreement with the ASBMB policy.

2.6. Immunofluorescence and Quantification of ZO-1 Deposits. Cells grown on coverslips were rinsed twice with PBS and fixed in cold methanol for $12 \mathrm{~min}$. After blockade with $\mathrm{PBS} / \mathrm{BSA}$ 5\% dilution for $60 \mathrm{~min}$ at RT and incubated for $90 \mathrm{~min}$ with anti-ZO-1 (ThermoFisher Scientific) and followed by $60 \mathrm{~min}$ of incubation with Alexa Fluor 488conjugated anti-rabbit IgG (Molecular Probes), cells were visualized on an FSX-100 (Olympus Life Science). Contrast, brightness, and focus settings were chosen so that all pixels were in the linear range. To quantify the average $\mathrm{ZO}-1$ length per cell, 4 fields were randomly selected, and the total length of ZO-1 in each field was outlined manually on Photoshop, followed by measurement using Image J software (NIH) [11-13]. Cell numbers were counted for each field with the DAPI Fluoromount-G (SouthernBiotech) slide mounting.

2.7. Statistical Analyses. Data were expressed as mean \pm 1 standard deviation (SD). One-way analysis of variance, Mann-Whitney, and Student $t$-test were appropriately performed, with a significant $p$ value set at 0.05 (MedCalc software).

\section{Results}

3.1. The Phosphorylation and Activation of AMPK Following a $\mathrm{Ca}^{2+}$ Switch Depend on CaMKK in MDCK Cells, Independently of $L K B 1$. Following a $\mathrm{Ca}^{2+}$ switch, we observed a mean 1.75-fold increase of pAMPK compared to S-MEM medium $(n=4, p<0.05)$ whereas total AMPK remained unchanged, as previously reported [11] (Figures 1(a) and 1(b)). Mean levels of pACC, a typical substrate of AMPK, followed a similar pattern, with a 5.3-fold increase following $\mathrm{Ca}^{2+}$ switch $(n=4, p<0.05)$. LKB1 and CaMKK are considered as the 2 major AMPK kinases [23]. In LKB1-shRNA MDCK cells, mean levels of pAMPK and pACC were 1.4-fold and 4.7-fold increase, respectively, in comparison to S-MEM $(n=4, p<0.05)$, with no significant difference with control Luc-shRNA ( $n=4$, not significant (ns)) (Figures 1(a) and 1(b)). Of important note, Luc-shRNA MDCK cells behave similarly as MDCK cells regarding AMPK phosphorylation/ activation and ZO- 1 relocation following $\mathrm{Ca}^{2+}$ switch $(n=4$, data not shown). Conversely, pharmacological inhibition of CaMKK using STO-609 prevented AMPK phosphorylation and activation after $\mathrm{Ca}^{2+}$ switch, with mean levels of pAMPK and pACC similar to S-MEM conditions $(n=4, \mathrm{~ns})$. Incubation of MDCK with AMPK inhibitor, dorsomorphin/compound $\mathrm{C}(50 \mu \mathrm{M})$ prevented AMPK autophosphorylation classically induced by $\mathrm{Ca}^{2+}$ switch (Figures $1(\mathrm{a})$ and $1(\mathrm{~b})$ ). These observations suggest that CaMKK plays a role in $\mathrm{Ca}^{2+}$-induced AMPK activation in MDCK cells, independently of LKB1.

3.2. Pharmacological Inhibitions of AMPK or CaMKK Prevent $\mathrm{Ca}^{2+}$-Induced TJ Relocation of ZO-1. During a $\mathrm{Ca}^{2+}$ switch, the translocation of $\mathrm{TJ}$-associated protein $\mathrm{ZO}-1$ from cell cytosol to cell-cell junctions represents a key and early step of TJ assembly [5]. Hence, we monitored the length of ZO-1 membrane deposits following a $\mathrm{Ca}^{2+}$ switch in MDCK cells exposed to various experimental conditions [11-13]. In normal conditions, readdition of $\mathrm{Ca}^{2+}$ causes a 4 -fold increase of ZO-1 length compared to S-MEM conditions (Figures $1(\mathrm{c})$ and $1(\mathrm{~d})$ ). After $2 \mathrm{~h}$ of $\mathrm{Ca}^{2+}$ switch, TJ were largely assembled as a classical chicken-wire network. In LKB1-shRNA MDCK cells, ZO-1 relocation followed a pattern similar to control MDCK $(n=4$, not significant). By contrast, pharmacological inhibition of CaMKK (using STO-609) $(n=4, p<0.05)$ or AMPK (dorsomorphin/compound C) $(n=4, p<0.05)$ prevented ZO-1 relocation induced by the $\mathrm{Ca}^{2+}$ switch (Figures $1(\mathrm{c})$ and $1(\mathrm{~d})$ ). These observations suggest that AMPK and CaMKK kinase activity participates in $\mathrm{Ca}^{2+}$-induced ZO-1 deposits in MDCK cells, independently of LKB1.

3.3. The Phosphorylation and Activation of AMPK in MDCK Cells Are Enhanced in the Presence of MSC, Which Is Associated with Faster Relocation of ZO-1 to Cell-Cell Contacts. TJ assembly in epithelial cells may be modulated by nonepithelial cells $[43,44]$. As an example, lymphocytes have been shown to boost $\mathrm{Ca}^{2+}$-induced activation of AMPK and accelerate TJ formation [43]. Similarly, we postulated that MSC may participate in TJ formation, and we investigated whether AMPK was implicated in such a process. In comparison to MDCK alone, phosphorylation and activation of AMPK was significantly increased in MDCK/MSC coculture, as demonstrated by mean levels of pAMPK/AMPK ratio $(n=4, p<0.05)$ and $\mathrm{pACC}(n=4, p<0.05)$ (Figures $2(\mathrm{a})$ and 2(b)). Of important note, immunoreactive signals for AMPK activation and pACC were undetectable in MSC alone, which suggest that only MDCK AMPK activation pathway is tested 


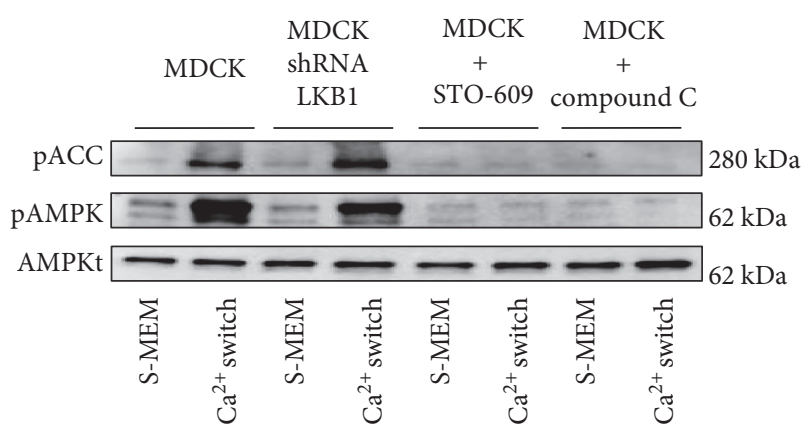

(a)

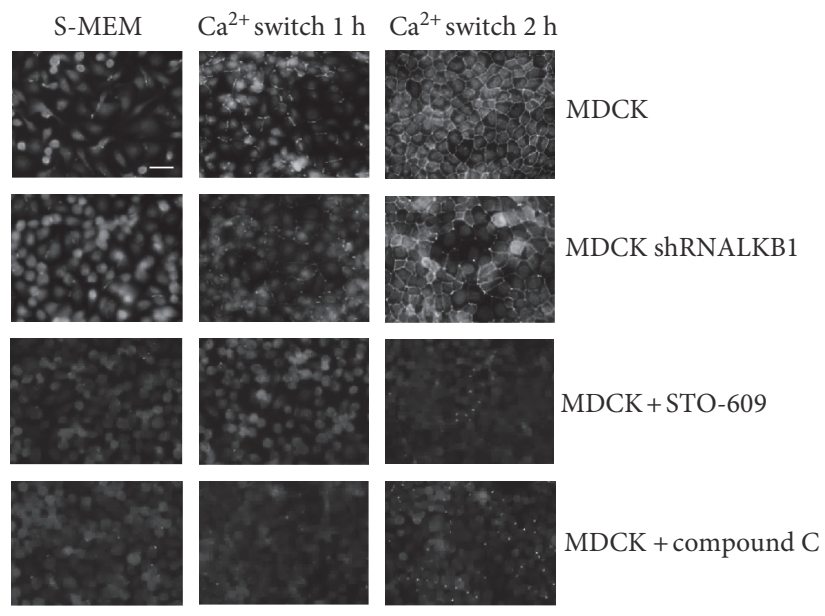

(c)
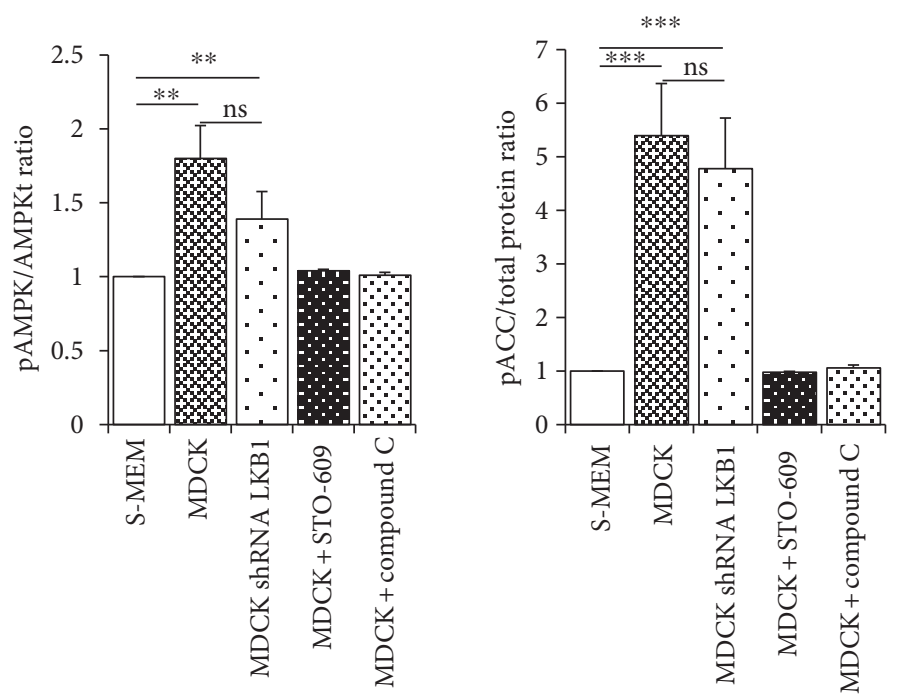

(b)

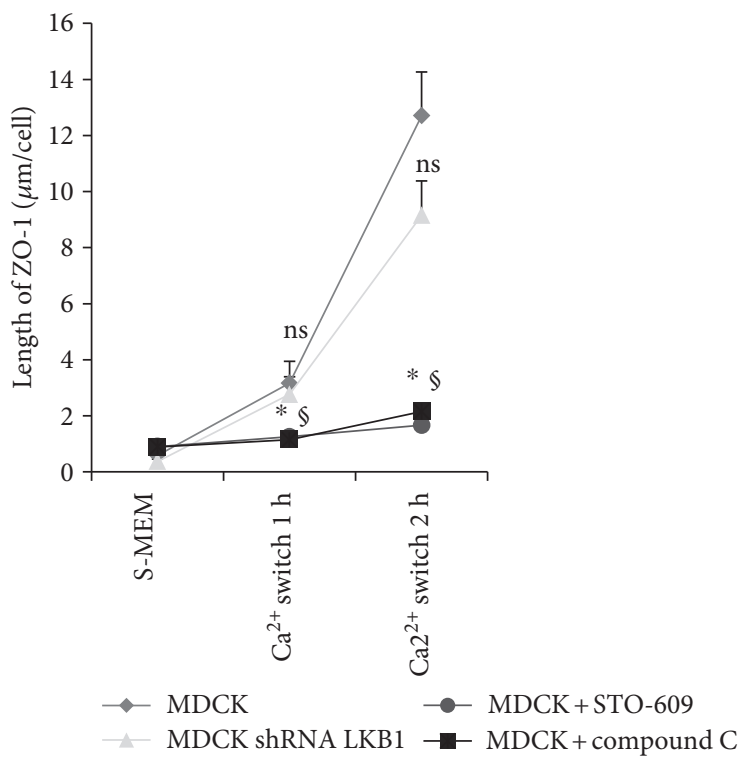

(d)

FIgUre 1: Role of the AMPK kinases, LKB1 and CaMKK, in AMPK activation and ZO-1 relocation following a Ca ${ }^{2+}$ switch in MDCK cells. Representative immunoblotting (a) and quantifications (b) of phospho-acetyl-Coa carboxylase (pACC), phospho-AMP-activated protein kinase (pAMPK), and total AMPK (AMPKt) in low $\mathrm{Ca}^{2+}$ conditions (S-MEM) and following Ca ${ }^{2+}$ switch using MDCK cells or LKB1shRNA MDCK cells. Compounds STO-609 and C were used as CaMKK and AMPK inhibitors, respectively. Quantifications of immunoreactive signals were performed by stain-free method after normalization to total protein content of each lane. Quantifications of phospho-ACC, phospho-AMPK, and AMPKt signals following $\mathrm{Ca}^{2+}$ switch were calculated and expressed by the ratio to the immunoreactive signal of SMEM condition in each individual experiment (a). For the sake of bar-graph clarity (b), SMEM values of all experiments were normalized to 1 in order to represent mean ratios of phospho-ACC/total protein content and phospho-AMPK/AMPKt in different experimental conditions (b). Data are presented as mean $\pm \mathrm{SD}$; ns: not significant, ${ }^{* *} p \leq 0.01,{ }^{* * *} p \leq 0.001$. Representative immunofluorescence (c) and quantifications (d) of ZO-1 deposits at increasing time points following $\mathrm{Ca}^{2+}$ switch in similar conditions as in (a) and (b) (scale bar: $16 \mu \mathrm{m}$ ). No statistically significant difference was observed between MDCK and LKB1-shRNA MDCK (ns: not significant). MDCK exposed to compound $\mathrm{C}\left({ }^{*} p \leq 0.01\right)$ or STO-609 $\left({ }^{\S} p \leq 0.01\right)$ showed a significant reduction of ZO-1 relocation in comparison to control MDCK. Data are presented as mean \pm SD.

in our model [45] (Supplementary Figure, panel B). In line with these observations, we monitored the time-course of ZO-1 relocation to cell-cell contacts following $\mathrm{Ca}^{2+}$ switch in the presence or absence of MSC (Figures 2(c) and 2(d)). After 16-hour deprivation of $\mathrm{Ca}^{2+}$, the length of TJ-associated
ZO-1 per cell was 3x higher in MDCK/MSC coculture compared to MDCK cells alone $(n=4, p<0.05)$ (Figures 2 (c) and $2(\mathrm{~d}))$. After $1 \mathrm{~h}$ of $\mathrm{Ca}^{2+}$ switch, $\mathrm{ZO}-1$ relocation to cell-cell contacts was twice higher in MDCK/MSC than in MDCK alone $(n=4, p<0.05)$. Still, at 2 hours post $\mathrm{Ca}^{2+}$ switch, the 


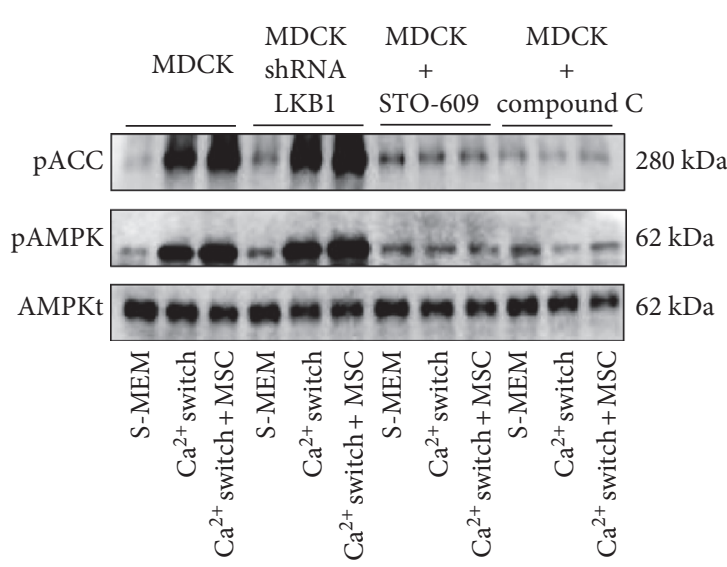

(a)

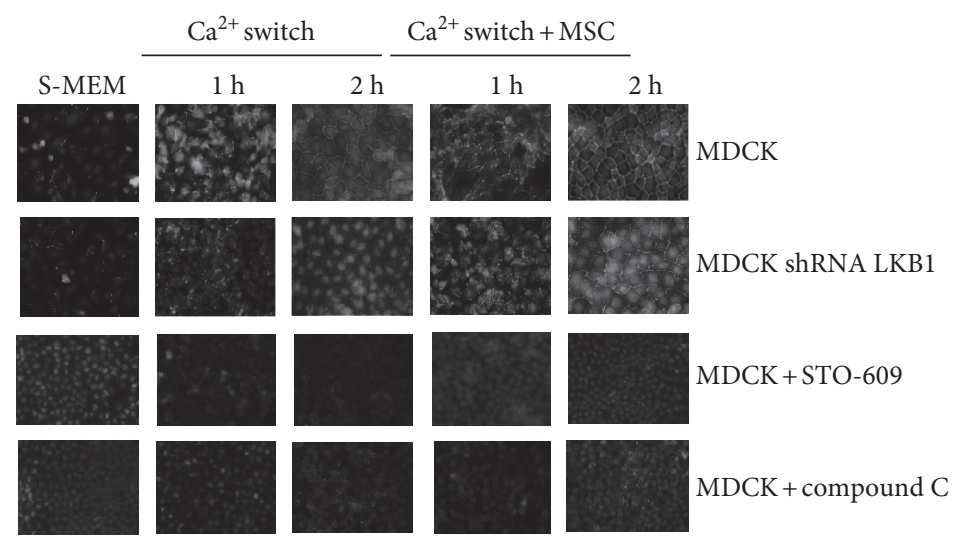

(c)
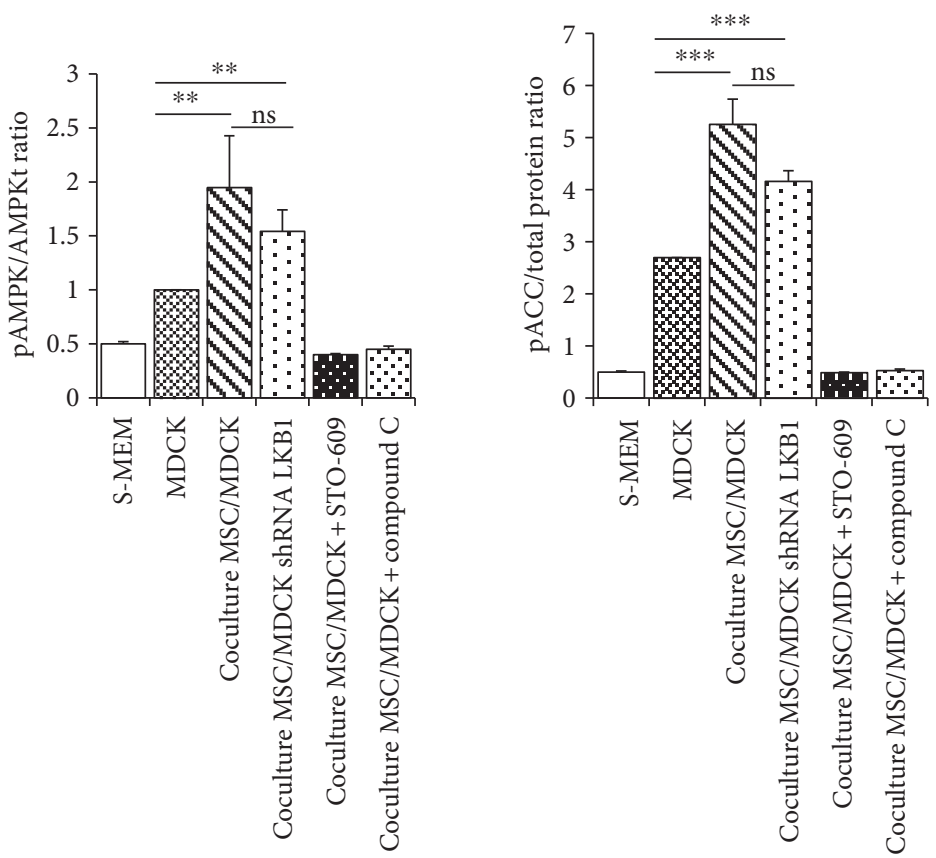

(b)

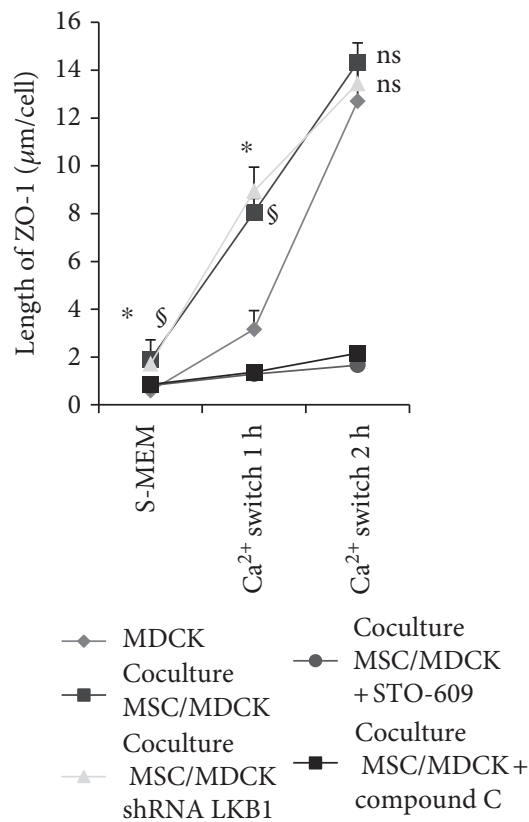

(d)

FIGURE 2: Impact of mesenchymal stromal cells (MSC) on AMPK activation and ZO-1 relocation following a Ca ${ }^{2+}$ switch in MDCK cells. Representative immunoblotting (a) and quantifications (b) of phospho-acetyl-Coa carboxylase (pACC), phospho-AMP-activated protein kinase (pAMPK), and total AMPK (AMPKt) in low $\mathrm{Ca}^{2+}$ conditions (S-MEM) and following Ca ${ }^{2+}$ switch using MDCK cells or LKB1shRNA MDCK cells, with versus without MSC. Compounds STO-609 and C were used as CaMKK and AMPK inhibitors, respectively. Quantifications of immunoreactive signals were performed by stain-free method after normalization to total protein content of each lane. Data are presented as mean $\pm \mathrm{SD}$; ns: not significant, ${ }^{* *} p \leq 0.01,{ }^{* * *} p \leq 0.001$. Representative immunofluorescence (c) and quantifications (d) of ZO-1 deposits at increasing time points following $\mathrm{Ca}^{2+}$ switch in similar conditions as in (a) and (b) (scale bar: $\left.16 \mu \mathrm{m}\right)$. MSC/ MDCK (i.e., MDCK $\left({ }^{\S} p \leq 0.01\right)$ or MDCK LKB1-shRNA $\left({ }^{*} p \leq 0.01\right)$ ) cocultures show significantly increased ZO-1 deposits at 1-hour post $\mathrm{Ca}^{2+}$ switch in comparison to MDCK alone. At 2 hours post $\mathrm{Ca}^{2+}$ switch, no significant (ns) difference in $\mathrm{ZO}-1$ lengths is observed between MDCK and MSC/MDCKs. Data are presented as mean \pm SD. 

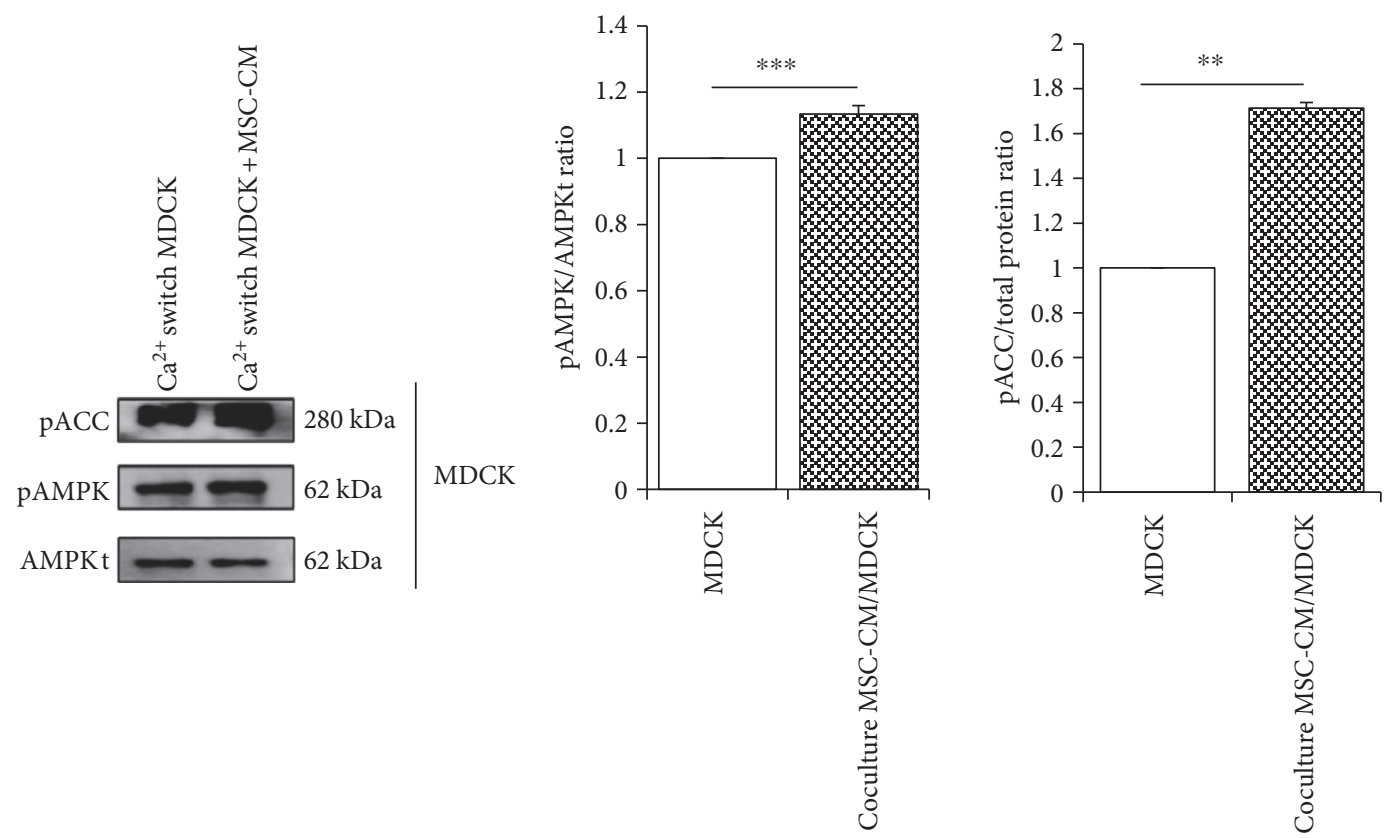

(a)

(b)

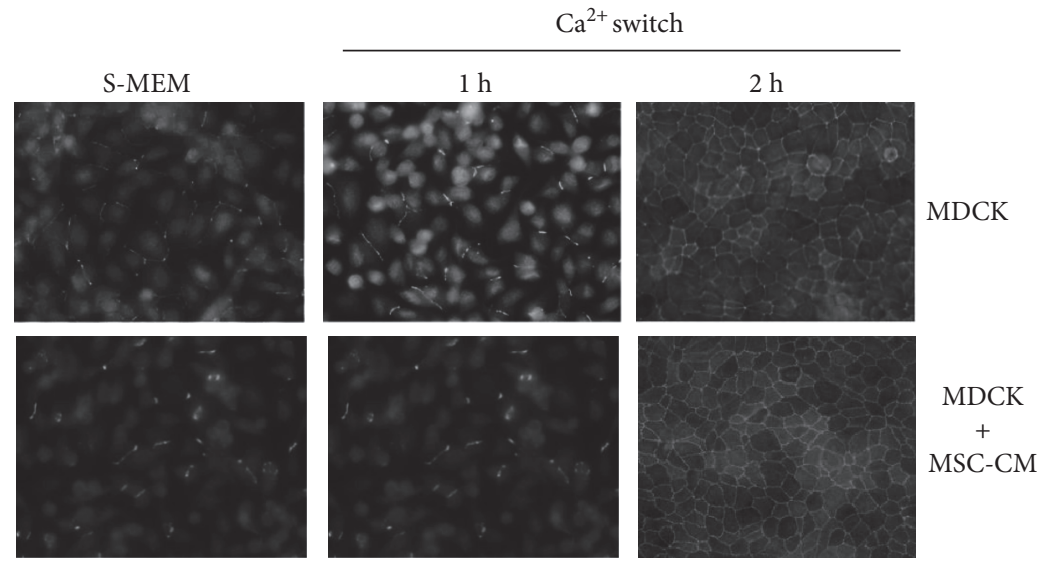

(c)

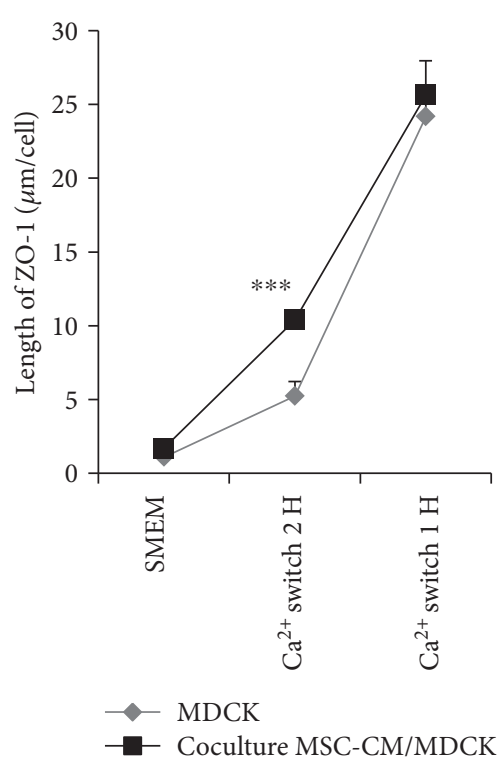

(d)

FIGURE 3: Impact of mesenchymal stromal cell- (MSC-) conditioned medium (CM) on AMPK activation and ZO-1 relocation following

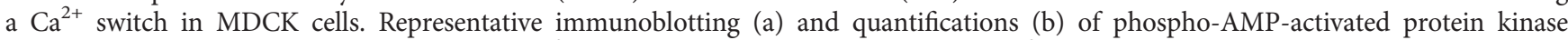
(pAMPK) and total AMPK (AMPKt) in low $\mathrm{Ca}^{2+}$ conditions (S-MEM) and following $\mathrm{Ca}^{2+}$ switch using MDCK cells exposed to regular versus MSC-preexposed medium. Quantifications of immunoreactive signals were performed by stain-free method after normalization to total protein content of each lane. Representative immunofluorescence (c) and quantifications (d) of ZO-1 deposits at increasing time points following $\mathrm{Ca}^{2+}$ switch in similar conditions as in (a) and (b) (scale bar: $16 \mu \mathrm{m}$ ). Data are presented as mean $\pm \mathrm{SD}$; ${ }^{* * *} p \leq 0.001$.

length of membrane-associated ZO-1 per cell was similar in both groups $(n=4, \mathrm{~ns})$ (Figures 2(c) and 2(d)). Of note, immunofluorescence signal for ZO-1 was undetectable in MSC cultured alone, which suggests that ZO-1 quantification only reflects ZO-1 deposits in MDCK cells in our model (Supplementary Figure, panel C). As a whole, these results indicate that MSC may accelerate ZO-1 deposition to cell-cell contacts at the time of TJ assembly in MDCK cells.

\subsection{MSC-Associated AMPK Activation and ZO-1 Relocation} in MDCK Cells Following $\mathrm{Ca}^{2+}$ Switch Are Prevented by $A M P K$ and CaMKK Inhibitors Independent of LKB1. Using LKB1-shRNA and Luc-shRNA MDCK cells cocultured with MSC, we assessed the role of LKB1 in MSC-enhanced AMPK activation and ZO-1 relocation. In LKB1-shRNA MDCK cells, mean levels of pAMPK/AMPK ratio $(n=4, p<0.05)$ and pACC $(n=4, p<0.05)$ were, respectively, 1.5 -fold increased and 2 -fold in the presence versus absence of 
MSC, to a similar extent of Luc-shRNA MDCK cells (Figures 2(a) and 2(b)). Furthermore, after a 16-hour deprivation of $\mathrm{Ca}^{2+}$, the length of $\mathrm{ZO}-1$ remaining at TJ sites per cell was $4 \mathrm{x}$ higher in MSC cocultured with LKB1-shRNA MDCK in comparison to MDCK alone $(n=4, p<0.05)$ (Figures 2(c) and 2(d)). After $1 \mathrm{~h}$ of $\mathrm{Ca}^{2+}$ switch, the length of ZO-1 membrane deposits per cell was twice longer in the presence versus absence of MSC $(n=4, p<0.05)$. In strong contrast, incubation of MSC/MDCK or MDCK cells alone with CaMKK (STO-609) or AMPK (dorsomorphin/compound C) inhibitors prevented MSC impact on AMPK activation and ZO-1 distribution, both after $\mathrm{Ca}^{2+}$ deprivation and $\mathrm{Ca}^{2+}$ switch (Figures 2(a) and 2(d)). These data suggest that, in a coculture system, MSC modulate $\mathrm{Ca}^{2+}$-induced CaMKK-mediated AMPK activation at the time of TJ assembly epithelial cells, independently of LKB1.

\subsection{MSC-Conditioned Culture Medium Slightly but} Significantly Enhances AMPK Activation and ZO-1 Relocation Following $\mathrm{Ca}^{2+}$ Switch in MDCK Cells. Mechanisms of MSC properties involve both direct cell-cell contacts and indirect impacts via paracrine factors [46]. To assess whether the impact of MSC on $\mathrm{Ca}^{2+}$-induced TJ assembly in MDCK cells requires direct cell-cell interactions, we performed the $\mathrm{Ca}^{2+}$ switch using an $\alpha$-MEM culture medium preexposed to MSC for 3 days. Hence, we observed that MSC-conditioned medium slightly (1.14-fold) but significantly $(n=4, p<0.05)$ increased AMPK phosphorylation. Thus, pACC was also increased by 1.7 -fold in the coculture $(n=4, p<0.05)$. The relocation of ZO- 1 was 1.7 -fold accelerated at 1 -hour post $\mathrm{Ca}^{2+}$ switch in comparison to untreated $\alpha$-MEM $(n=4, p<0.05)$ (Figures 3(a) and 3(d)). There was no difference between MSC-conditioned and untreated $\alpha$ MEM at 2 hours post $\mathrm{Ca}^{2+}$ switch (Figures 3(b) and 3(c)).

\section{Discussion}

The present in vitro observations suggest that bone marrow-derived MSC modulate epithelial TJ at the time of their $\mathrm{Ca}^{2+}$-induced assembly. The relocation of TJassociated adaptor protein, ZO-1, to MDCK cell-cell contacts was indeed significantly accelerated in the presence of MSC. Furthermore, AMPK phosphorylation and activation at the time of $\mathrm{Ca}^{2+}$-induced epithelial $\mathrm{TJ}$ assembly were significantly enhanced when MDCK cells were cocultured with MSC, which could be prevented by the pharmacological inhibition of CaMKK. Conversely, the depletion of LKB1 did not significantly influence AMPK phosphorylation following $\mathrm{Ca}^{2+}$ switch, with or without MSC coculture.

AMPK activity is modulated by 2 major upstream kinases, that is, LKB1 and CaMKK [18-20, 47]. Still, the respective contribution of each of these AMPK kinases in AMPK activation at the time of a $\mathrm{Ca}^{2+}$ switch remains unknown $[11,18]$. LKB1 provides a high basal level of AMPK phosphorylation, which is modulated by the binding of AMP to the AMPK $\gamma$-subunit. AMP binding to the $\gamma$-subunit allosterically activates AMPK, making it more susceptible for phosphorylation of the $\alpha$-subunit activation loop (at residue Thr172) by LKB1 [48]. Note that AMPK activation associated with $\mathrm{Ca}^{2+}$-induced TJ assembly is independent of changes in AMP/ATP ratio or energy privation [11, 18]. Conversely, CaMKK kinase has been shown to trigger AMPK phosphorylation on Thr172 in response to increased intracellular $\mathrm{Ca}^{2+}$ concentration with no necessary changes in AMP or ADP levels [21, 49]. Our present in vitro observations further support a role for CaMKK in the activation of AMPK during a $\mathrm{Ca}^{2+}$ switch, independently of LKB1 activity. Hence, the pharmacological inhibition of CaMKK hampered AMPK phosphorylation and ZO-1 relocation when culture conditions were shifted from low to high $\mathrm{Ca}^{2+}$ concentration, whereas the inactivation of LKB1 did not significantly influence these processes.

Circulating factors and cells have been shown to modulate TJ formation and maintenance in epithelia $[50,51]$. Hence, lymphocytes accelerate TJ assembly in a coculture in vitro model compared to epithelial cells alone [43]. This acceleration was found to be mediated by AMPK, independently of changes in cellular ATP levels. Furthermore, it was found to be activated by the proinflammatory cytokine TNF-alpha [43]. In line with these observations, coculturing endometrial epithelial cells with peripheral blood leukocytes improves both the survival of leukocytes and the epithelial barrier function, as reflected by a 4 -fold increase in the transepithelial resistance as compared to epithelial cells alone [44]. In this study, direct cell-cell contacts were required for the beneficial impact of immune cells. In our model, we hypothesized that MSC may also influence TJ of epithelial cells given the previous reports about their tissue-repair properties in various organs and tissues [31, 32, 52, 53]. MSC effects are known to be mediated by both direct cell-cell contacts and paracrine secretion of MVs $[54,55]$. Using a classical model of $\mathrm{Ca}^{2+}$-induced TJ assembly [8], we found that the presence of MSC was associated with a significantly faster deposition of ZO-1 to cell-cell contacts. Furthermore, MSC influence was abrogated in case of cell incubation with CaMKK or AMPK inhibitors, suggesting a key role of the AMPK pathway in such a process. Of important note, AMPKdependent and independent roles of compound $\mathrm{C}$ have been reported in a context-dependent manner [56].

These observations could be partly reproduced by incubating epithelial cells with MSC-conditioned medium, which supports a fractional role for MSC-derived MVs in epithelial TJ regulation.

\section{Conclusion}

As a whole, we report on the role of CaMKK as AMPK kinase at the time of $\mathrm{Ca}^{2+}$-induced assembly of epithelial TJ, independently of LKB1. Moreover, we highlight the impact of MSC in the AMPK-mediated regulation of epithelial TJ, via both direct cell-cell contacts and MSC-derivated particles and MSC-derived MVs. These findings open novel research avenues in the deciphering of MSC repair properties.

\section{Conflicts of Interest}

All the authors declared that there is no conflict of interest regarding the publication of this paper. 


\section{Acknowledgments}

P. Erpicum and F. Jouret are fellows of the Fonds National de la Recherche Scientifique (FNRS) and received support from the University of Liège (Fonds Spéciaux à la Recherche and Fonds Léon Fredericq) and the FNRS (research credits 2013 and 2016).

\section{References}

[1] M. J. Caplan, P. Seo-Mayer, and L. Zhang, "Epithelial junctions and polarity: complexes and kinases," Current Opinion in Nephrology and Hypertension, vol. 17, no. 5, pp. 506-512, 2008.

[2] J. Hou, "The kidney tight junction (review)," International Journal of Molecular Medicine, vol. 34, no. 6, pp. 1451-1457, 2014.

[3] L. Gonzalezmariscal, "Tight junction proteins," Progress in Biophysics and Molecular Biology, vol. 81, no. 1, pp. 1-44, 2003.

[4] E. McNeil, C. T. Capaldo, and I. G. Macara, "Zonula occludens-1 function in the assembly of tight junctions in Madin-Darby canine kidney epithelial cells," Molecular Biology of the Cell, vol. 17, no. 4, pp. 1922-1932, 2006.

[5] A. S. Fanning and J. M. Anderson, "Zonula occludens-1 and -2 are cytosolic scaffolds that regulate the assembly of cellular junctions," Annals of the New York Academy of Sciences, vol. 1165, no. 1, pp. 113-120, 2009.

[6] R. G. Contreras, J. H. Miller, M. Zamora, L. Gonzalez-Mariscal, and M. Cereijido, "Interaction of calcium with plasma membrane of epithelial (MDCK) cells during junction formation," The American Journal of Physiology, vol. 263, no. 2, Part 1, pp. C313-C318, 1992.

[7] L. Gonzalez-Mariscal, B. Chávez de Ramírez, and M. Cereijido, "Tight junction formation in cultured epithelial cells (MDCK)," The Journal of Membrane Biology, vol. 86, no. 2, pp. 113-125, 1985.

[8] J. Meldolesi, G. Castiglioni, R. Parma, N. Nassivera, and P. De Camilli, "Ca++-dependent disassembly and reassembly of occluding junctions in guinea pig pancreatic acinar cells. Effect of drugs," The Journal of Cell Biology, vol. 79, no. 1, pp. 156$172,1978$.

[9] C. E. Palant, M. E. Duffey, B. K. Mookerjee, S. Ho, and C. J. Bentzel, "Ca2+ regulation of tight-junction permeability and structure in Necturus gallbladder," The American Journal of Physiology, vol. 245, no. 3, pp. C203-C212, 1983.

[10] L. Gonzalez-Mariscal, R. G. Contreras, J. J. Bolívar, A. Ponce, B. Chávez De Ramirez, and M. Cereijido, "Role of calcium in tight junction formation between epithelial cells," The American Journal of Physiology, vol. 259, no. 6, Part 1, pp. C978-C986, 1990.

[11] L. Zhang, J. Li, L. H. Young, and M. J. Caplan, "AMP-activated protein kinase regulates the assembly of epithelial tight junctions," Proceedings of the National Academy of Sciences of the United States of America, vol. 103, no. 46, pp. 17272-17277, 2006.

[12] L. Zhang, F. Jouret, J. Rinehart et al., "AMP-activated protein kinase (AMPK) activation and glycogen synthase kinase- $3 \beta$ (GSK-3 $\beta$ ) inhibition induce Ca2+-independent deposition of tight junction components at the plasma membrane," The Journal of Biological Chemistry, vol. 286, no. 19, pp. 1687916890, 2011.
[13] F. Jouret, J. Wu, M. Hull et al., "Activation of the $\mathrm{Ca}(2)+-$ sensing receptor induces deposition of tight junction components to the epithelial cell plasma membrane," Journal of Cell Science, vol. 126, Part 22, pp. 5132-5142, 2013.

[14] J. Chen and M. Zhang, "The Par3/Par6/aPKC complex and epithelial cell polarity," Experimental Cell Research, vol. 319, no. 10, pp. 1357-1364, 2013.

[15] N. Aznar, A. Patel, C. C. Rohena et al., “AMP-activated protein kinase fortifies epithelial tight junctions during energetic stress via its effector GIV/Girdin," eLife, vol. 5, article e71, 2016.

[16] J. H. Lee, H. Koh, M. Kim et al., "Energy-dependent regulation of cell structure by AMP-activated protein kinase," Nature, vol. 447, no. 7147, pp. 1017-1020, 2007.

[17] D. G. Hardie, "AMP-activated protein kinase: an energy sensor that regulates all aspects of cell function," Genes \& Development, vol. 25, no. 18, pp. 1895-1908, 2011.

[18] B. Zheng and L. C. Cantley, "Regulation of epithelial tight junction assembly and disassembly by AMP-activated protein kinase," Proceedings of the National Academy of Sciences of the United States of America, vol. 104, no. 3, pp. 819-822, 2007.

[19] R. J. Shaw, M. Kosmatka, N. Bardeesy et al., "The tumor suppressor LKB1 kinase directly activates AMP-activated kinase and regulates apoptosis in response to energy stress," Proceedings of the National Academy of Sciences of the United States of America, vol. 101, no. 10, pp. 3329-3335, 2004.

[20] S. A. Hawley, D. A. Pan, K. J. Mustard et al., "Calmodulindependent protein kinase kinase- $\beta$ is an alternative upstream kinase for AMP-activated protein kinase," Cell Metabolism, vol. 2, no. 1, pp. 9-19, 2005.

[21] D. G. Hardie, F. A. Ross, and S. A. Hawley, "AMPK: a nutrient and energy sensor that maintains energy homeostasis," Nature Reviews. Molecular Cell Biology, vol. 13, no. 4, pp. 251-262, 2012.

[22] M. Sebbagh, S. Olschwang, M.-J. Santoni, and J.-P. Borg, "The LKB1 complex-AMPK pathway: the tree that hides the forest," Familial Cancer, vol. 10, no. 3, pp. 415-424, 2011.

[23] D. Carling, M. J. Sanders, and A. Woods, "The regulation of AMP-activated protein kinase by upstream kinases," International Journal of Obesity, vol. 32, pp. S55-S59, 2008.

[24] M. Sebbagh, M.-J. Santoni, B. Hall, J.-P. Borg, and M. A. Schwartz, "Regulation of LKB1/STRAD localization and function by E-cadherin," Current Biology, vol. 19, no. 1, pp. 37-42, 2009.

[25] Y. Oligschlaeger, M. Miglianico, D. Chanda et al., "The recruitment of AMP-activated protein kinase to glycogen is regulated by autophosphorylation," The Journal of Biological Chemistry, vol. 290, no. 18, 2015.

[26] T. Ooshio, R. Kobayashi, W. Ikeda et al., "Involvement of the interaction of afadin with ZO-1 in the formation of tight junctions in Madin-Darby canine kidney cells," The Journal of Biological Chemistry, vol. 285, no. 7, pp. 50035012, 2010.

[27] D. Castanares-Zapatero, C. Bouleti, C. Sommereyns et al., "Connection between cardiac vascular permeability, myocardial edema, and inflammation during sepsis," Critical Care Medicine, vol. 41, no. 12, pp. e411-e422, 2013.

[28] P. W. Seo-Mayer, G. Thulin, L. Zhang et al., "Preactivation of AMPK by metformin may ameliorate the epithelial cell damage caused by renal ischemia," American Journal of Physiology. Renal Physiology, vol. 301, no. 6, pp. F1346F1357, 2011. 
[29] J. Lempiäinen, P. Finckenberg, J. Levijoki, and E. Mervaala, "AMPK activator AICAR ameliorates ischaemia reperfusion injury in the rat kidney," British Journal of Pharmacology, vol. 166, no. 6, pp. 1905-1915, 2012.

[30] V. Cantaluppi, L. Biancone, A. Quercia, M. C. Deregibus, G. Segoloni, and G. Camussi, "Rationale of mesenchymal stem cell therapy in kidney injury," American Journal of Kidney Diseases, vol. 61, no. 2, pp. 300-309, 2013.

[31] P. Erpicum, O. Detry, L. Weekers et al., "Mesenchymal stromal cell therapy in conditions of renal ischaemia/reperfusion," Nephrology Dialysis Transplantation, vol. 29, no. 8, pp. 14871493, 2014, Oxford University Press.

[32] P. Rowart, P. Erpicum, O. Detry et al., "Mesenchymal stromal cell therapy in ischemia/reperfusion injury," Journal of Immunology Research, vol. 2015, Article ID 602597, 8 pages, 2015.

[33] M. Franquesa, M. J. Hoogduijn, M. E. Reinders et al., "Mesenchymal stem cells in solid organ transplantation (MiSOT) fourth meeting: lessons learned from first clinical trials," Transplantation, vol. 96, no. 3, pp. 234-238, 2013.

[34] N. Souidi, M. Stolk, and M. Seifert, "Ischemia-reperfusion injury: beneficial effects of mesenchymal stromal cells," Current Opinion in Organ Transplantation, vol. 18, no. 1, pp. 34-43, 2013.

[35] S. Winkler, M. Hempel, S. Brückner, H.-M. Tautenhahn, R. Kaufmann, and B. Christ, "Identification of pathways in liver repair potentially targeted by secretory proteins from human mesenchymal stem cells," International Journal of Molecular Sciences, vol. 17, no. 7, p. 1099, 2016.

[36] W. Broekman, G. D. Amatngalim, Y. de Mooij-Eijk et al., "TNF-alpha and IL-1beta-activated human mesenchymal stromal cells increase airway epithelial wound healing in vitro via activation of the epidermal growth factor receptor," Respiratory Research, vol. 17, no. 1, p. 3, 2016.

[37] K. M. Akram, S. Samad, M. A. Spiteri, and N. R. Forsyth, "Mesenchymal stem cells promote alveolar epithelial cell wound repair in vitro through distinct migratory and paracrine mechanisms," Respiratory Research, vol. 14, no. 1, p. 9, 2013.

[38] A. I. Caplan and J. E. Dennis, "Mesenchymal stem cells as trophic mediators," Journal of Cellular Biochemistry, vol. 98, no. 5, pp. 1076-1084, 2006.

[39] G. Raposo and W. Stoorvogel, "Extracellular vesicles: exosomes, microvesicles, and friends," The Journal of Cell Biology, vol. 200, no. 4, pp. 373-383, 2013.

[40] G. Camussi, M. C. Deregibus, S. Bruno, V. Cantaluppi, and L. Biancone, "Exosomes/microvesicles as a mechanism of cell-tocell communication," Kidney International, vol. 78, no. 9, pp. 838-848, 2010.

[41] J. V. Bonventre, "Microvesicles from mesenchymal stromal cells protect against acute kidney injury," Journal of the American Society of Nephrology, vol. 20, no. 5, pp. 927-928, 2009.

[42] M. Dominici, K. Le Blanc, I. Mueller et al., "Minimal criteria for defining multipotent mesenchymal stromal cells. The International Society for Cellular Therapy position statement," Cytotherapy, vol. 8, no. 4, pp. 315-317, 2006.

[43] X. X. Tang, H. Chen, S. Yu, L. Zhang, M. J. Caplan, and H. C. Chan, "Lymphocytes accelerate epithelial tight junction assembly: role of AMP-activated protein kinase (AMPK)," PloS One, vol. 5, no. 8, article e12343, 2010.

[44] L. Ho, L. Tsang, Y. Chung, and H. Chan, "Establishment of a mouse primary co-culture of endometrial epithelial cells and peripheral blood leukocytes: effect on epithelial barrier function and leukocyte survival," Cell Biology International, vol. 30, no. 12, pp. 977-982, 2006.

[45] C. De Meester, A. D. Timmermans, M. Balteau et al., "Role of AMP-activated protein kinase in regulating hypoxic survival and proliferation of mesenchymal stem cells," Cardiovascular Research, vol. 101, no. 1, pp. 20-29, 2014.

[46] X.-Y. Zhu, V. Urbieta-Caceres, J. D. Krier, S. C. Textor, A. Lerman, and L. O. Lerman, "Mesenchymal stem cells and endothelial progenitor cells decrease renal injury in experimental swine renal artery stenosis through different mechanisms," Stem Cells, vol. 31, no. 1, pp. 117-125, 2013.

[47] D. Hardie and S. Hawley, "AMP-activated protein kinase: the energy charge hypothesis revisited," BioEssays, vol. 23, no. 12, pp. 1112-1119, 2001.

[48] G. R. Steinberg and B. E. Kemp, "AMPK in health and disease," Physiological Reviews, vol. 89, no. 3, pp. 10251078, 2009.

[49] C. Cantó and J. Auwerx, "AMP-activated protein kinase and its downstream transcriptional pathways," Cellular and Molecular Life Sciences, vol. 67, no. 20, pp. 3407-3423, 2010.

[50] J. D. Matthews, C. M. Weight, and C. A. Parkos, "Leukocyteepithelial interactions and mucosal homeostasis," Toxicologic Pathology, vol. 42, no. 1, pp. 91-98, 2014.

[51] K. Bäsler and J. M. Brandner, "Tight junctions in skin inflammation," Pflügers Archiv-European Journal of Physiology, vol. 1, no. 1, pp. 3-14, 2016.

[52] A. M. Dimarino, A. I. Caplan, and T. L. Bonfield, "Mesenchymal stem cells in tissue repair," Frontiers in Immunology, vol. 4, p. 201, 2013.

[53] M. Morigi and A. Benigni, "Mesenchymal stem cells and kidney repair," Nephrology, Dialysis, Transplantation, vol. 28, no. 4, pp. 788-793, 2013.

[54] L. Biancone, S. Bruno, M. C. Deregibus, C. Tetta, and G. Camussi, "Therapeutic potential of mesenchymal stem cellderived microvesicles," Nephrology, Dialysis, Transplantation, vol. 27, no. 8, pp. 3037-3042, 2012.

[55] J. He, Y. Wang, S. Sun et al., "Bone marrow stem cellsderived microvesicles protect against renal injury in the mouse remnant kidney model," Nephrology (Carlton, Vic.), vol. 17, no. 5, pp. 493-500, 2012.

[56] E. Rao, Y. Zhang, Q. Li et al., "AMPK-dependent and independent effects of AICAR and compound C on T-cell responses," Oncotarget, vol. 7, no. 23, pp. 33783-33795, 2014. 

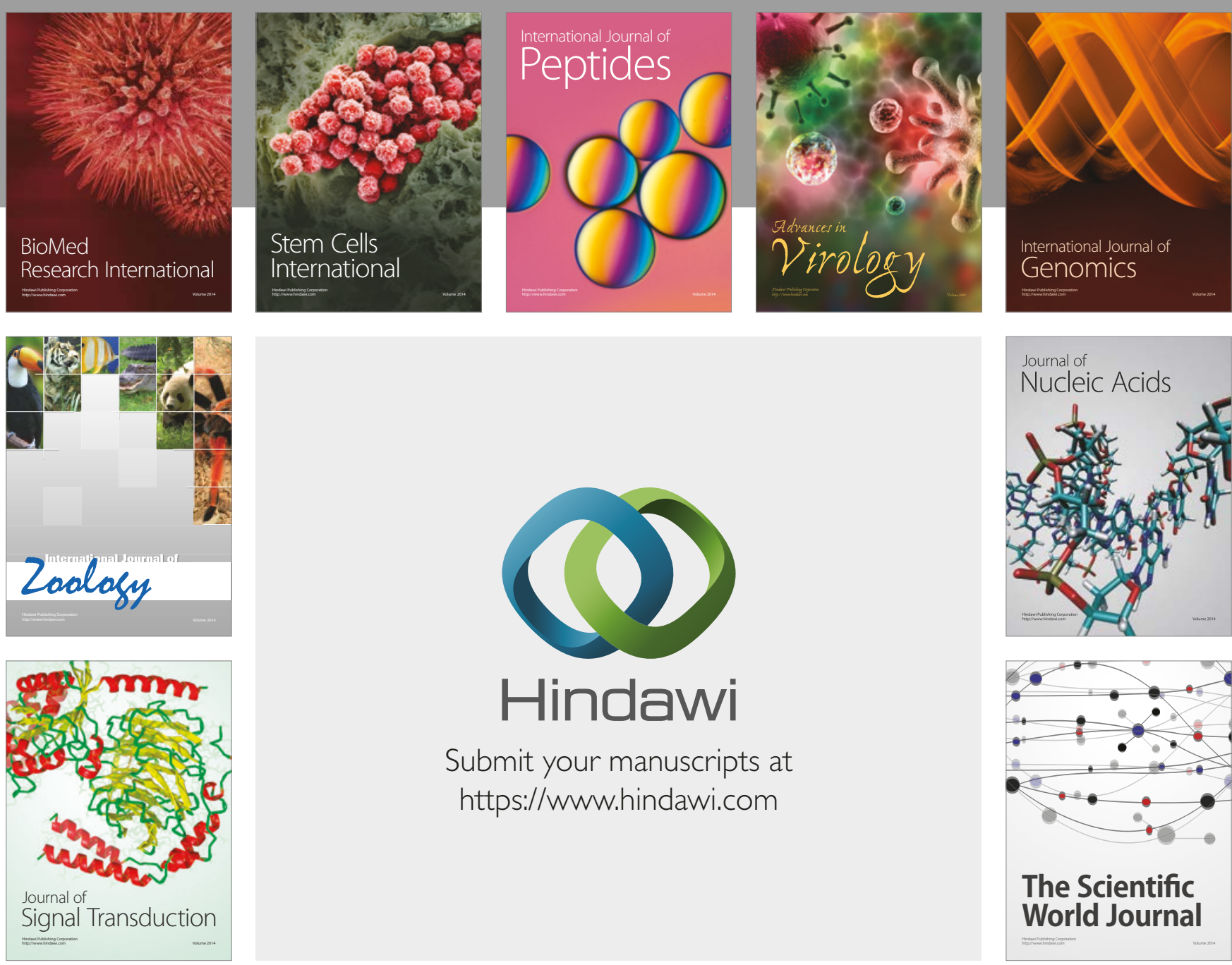

Submit your manuscripts at

https://www.hindawi.com
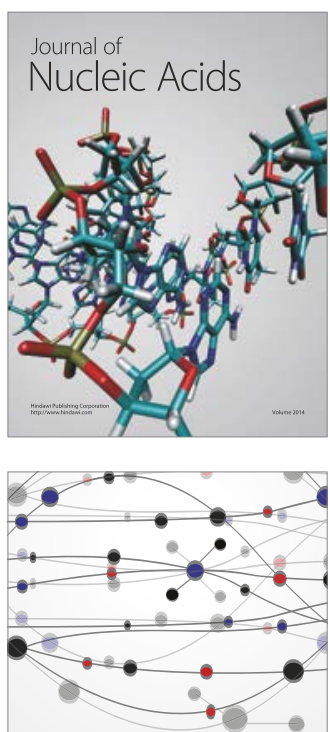

The Scientific World Journal

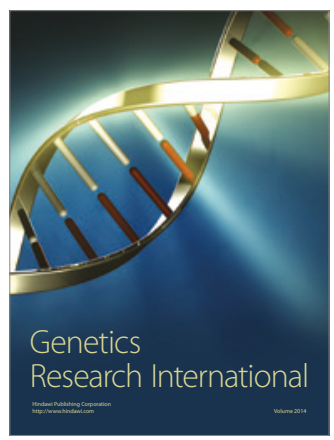

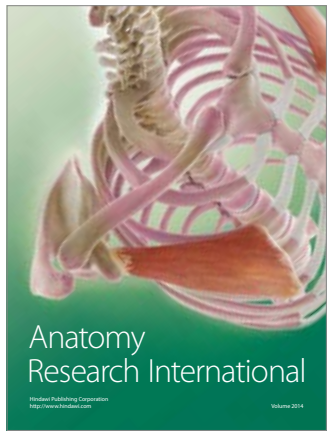

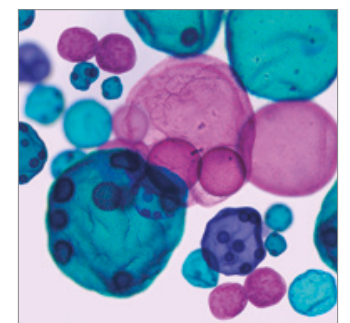

International Journal of Microbiology
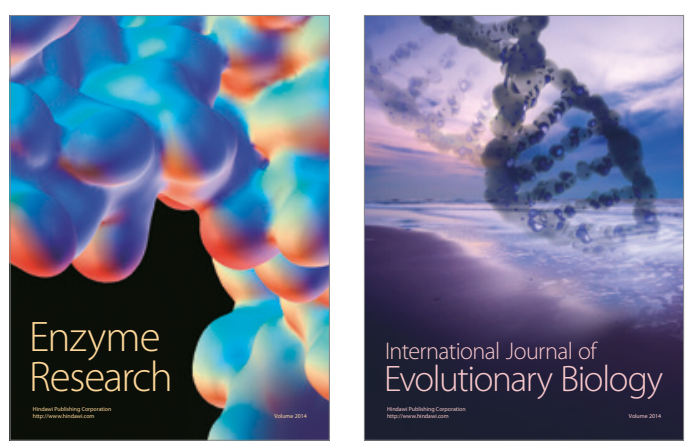
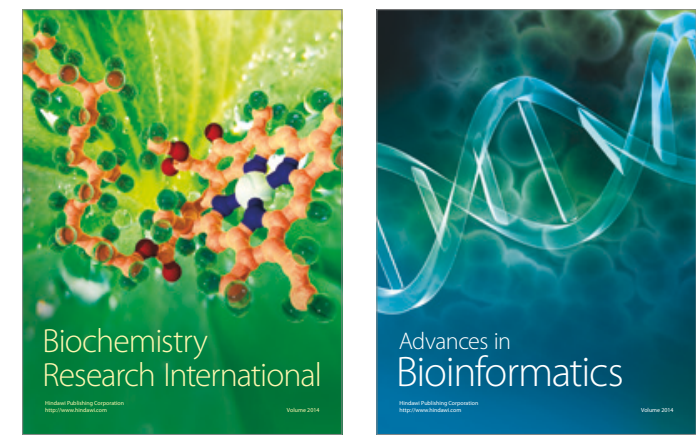

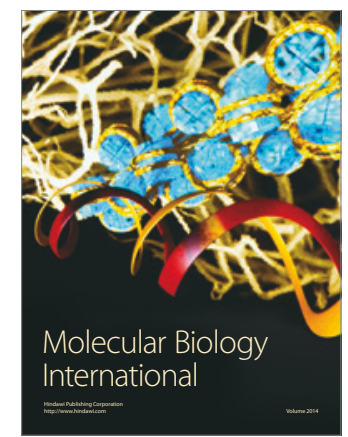

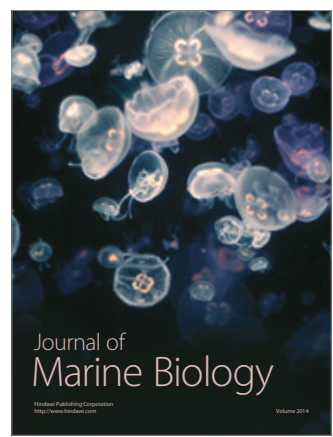

